\title{
Adela MUNTEAN \\ Spherical encounters of the Anthropocene: \\ from Telescope to Kaleidoscope
}

\begin{abstract}
Spherical encounters of the Anthropocene refers to the impact of humanity's global ecological footprint that is addressed through the metaphor of the spheres. The new human-generated AnthropoNoo-spheres and their interaction with the Spheres of Earth are tackled through ecocritical investigation showcasing the diverse representational modalities and intermedial expressions found in the Planetarium and artistic spherical environments. The technological requirements for high quality geoscientific visualizations have taken about a decade longer to mature than the astronomical features of digital planetariums, but Earth System Science is finally adopted by the Planetarium and artists.

From Telescope to Kaleidoscope refers to the transition between the emergence of the modern scientific, telescopic viewpoint, and the increasing rationalization and bureaucratization of society enabled by the technological and economic advances of the age, that created a sense of alienation of the individual, from the natural environment and the social other, to an environmental re-enchantment, a regaining of our sense of wonder and awe brought back by the kaleidoscopic visions artists apply to these spherical mediums. As this article seeks to demonstrate, the scientific and imaginative visions fused into interconnected, parallel universes can challenge our mundane notions opening up new causal connections, creating exciting discoveries, all under the notion of Anthropocene. However, these spherical environments showcasing kaleidoscopic visions are not an aestheticization of the Anthroposphere, but a testimonv that artists as antennas capture and showcase the contemporary human
\end{abstract} sentiment, topics and observations by conveying experiential projects which involve strategies to forge communities, to re-build lost paradises, to re-connect us with the sky and earth, to foster regeneration and new sustainable systems and to ask future questions. Through the stylistic element of the Cosmic Zoom, we are travelling from the unknown of the outer space to the inner reaches of our human heart.

Keywords: sphairopoiia (the art of sphere-making), enviromental reenchantment of the world, overview effect, liminoid, cosmic zoom, dome, anthroposphere, sphere interactions..

\section{Adela MUNTEAN}

Babes-Bolyai University, Cluj-Napoca adelamuntean.com@gmail.com

EKPHRASIS, 2/2020

THE ANTHROPOCENE AND INTERMEDIAL ECOCRITICISM pp. $144-166$

DOI: $10.24193 /$ ekphrasis. 24.8

Published First Online: 14 December 2020 


\section{The Dome as Sky}

In 1924, a new type of construction appeared on the surface of the earth on the roof of the Zeiss factory in Jena, a small city in Eastern Germany: a hemisphere 16 meters in diameter was representing the very first geodesic dome made solely of interlinking rods. On the outside, the dome was plastered with a thin layer of cement and inside the "white interior was used as a projection surface for a multitude of projection apparatuses arranged at the center of the construction, so that the images produced were showing the visible stars in their position and movement, just as we were accustomed to see them outside in nature” (Bauersfeld quoted in Firebrace 59). Walter Bauersfeld invented not only the mechanics of a new type of projection system, but a new way of looking at celestial bodies moving across the sky and a new building typology. The dome architecture was imitating the shape of the sky as envisioned from ancestral times. Through its obvious relationship to the perceived curvature of the firmament, the dome provides a metaphorical bridge between finite sensory experience and the infinite mythic imagination (Lambert 15). By the magic of this apparatus, humanity at last achieved its ancient quest of enclosing the universe inside a room.

The newly invented projection system imitated the tilt and rotation of the earth: with six classes of brightness simulating 4,500 stars and multiple projecting globes placed on a metal rod set at 23 degrees to the horizontal, representing the tilt of the earth, the sun, and moon, and the five planets were also represented through light. The whole projection mechanism could swivel to imitate the turning of the earth at various speeds passing a year in fifty seconds, two minutes or four minutes (Firebrace 62). The later added refinements to the projection system called Zeiss Mark II made it possible to reveal the positions of the planets and stars as seen from any latitude and in any year in the past and future. The Planetarium as a time-space machine was thus established. This new screen type and projection system intimately followed the mechanism of Nature at a level beyond human perception.

The important condition that made possible and necessary the projection Planetarium was the invention of electricity: Oskar von Miller, the founder of the Deutsches Museum in Munich, who commissioned Walter Baursfeld to create the Planetarium, was also director of Bavaria's largest electricity company, AEG, and had been responsible for late nineteenthcentury technical progress in building distribution systems for electrical power, thus bringing electricity to German towns. He saw electricity not just as a source of power, but also as a civilizing force (Firebrace 54). Thus, the anthropocenic moment in astronomy can be traced back to the invention of electricity which cut our ontological connection with the stars, so that an optical link to the cosmos was created and the real sky experience was replaced by a complex media simulation that is the Planetarium itself: "Somewhere in the whole Miller enterprise, there was a curious contradiction, which would continue to run through stories of the creation of simulations of natural phenomena. The glare of electrical city lighting, which 
Miller himself had brought to German cities, made the stars difficult for city-dwellers to see, so an artificial sky was now necessary as a replacement for the real experience. The natural world, no longer fully visible due to the advances of lighting technology, would be replaced by an ingenious simulation" (54). This ontological link with the sky that suddenly shifted was commented on by various critics including Walter Benjamin. To the Planetarium, the last section of his book entitled One-way-Street, was added at the last moment, shortly before the publication of his book, the Berlin Planetarium opened. He formulated the question of a purely optical link to the cosmos and the replacement of the actual experience by simulation: can the ancients' connection with the cosmos, the ecstatic trance Rausch (Firebrace 75-76), the deep human feeling of being part of the universe communally achieved, be satisfied by an artificial recreation?

\section{The Earth as a medium for science and art}

In 1960, the United States put its first earth-observing environmental satellite into orbit around the planet. Over the decades, these satellites have provided invaluable information, and the vantage point of space has provided new perspectives on earth. In 2012, NASA created a book entitled Earth as Art that celebrates earth's aesthetic beauty in the patterns, shapes, colors, and textures of the land, oceans, ice, and atmosphere. The book features 75 stunning images of earth from the Terra, Landsat 5, Landsat 7, EO-1, and Aqua satellites. Sensors on these satellites can measure light outside of the visible range, so the images show more than what is visible to the naked eye. The images are intended for viewing enjoyment rather than scientific interpretation (NASA website). Since the launch of Explorer 1 in 1958 to study cosmic radiation, earth observing systems have enabled scientists to investigate the complex relationships between human activities and earth's ecosystems. The expanding global network of satellites has played a crucial role in studying previously invisible interconnections by enabling new forms of "planetary proprioception" (Barasch and Fedorova quoted in McConville 296). Consequently, with the photographs taken from space toward the earth naming Earthrise (1968), "the most influential environmental photograph ever taken”; Blue Marble (1972), Pale Blue Dot (1990), a reverse gaze was created forming humanity's 'cosmic horizon'. After years of observing the sky from the earth, the earth gets observed from the sky, a reverse gaze made possible by satellites marking our second major anthropocenic moment when the earth became officially a medium for science and art starting a new era that put in focus humanity's role in the Cosmos. Earth Day was celebrated for the first time in 1970 to demonstrate support for environmental protection; the art movement Earth art, now currently called Land art, also emerged in that period when spiritual yearnings concerning the planet earth as home to mankind 
were on the agenda (Atkins 2013). As earth "lacked" an operating manual, in 1969 Fuller published Operating Manual for Spaceship Earth (1969/2008a), a foundational work for the contemporary environmental movement which became a major intellectual touchstone for environmental actions and the green movement's history (Boulding 1966). The book relates the idea that the earth is a spaceship, with the sun as our energy supplier. "We are all astronauts" says Fuller. The idea of the earth is that of a mechanical vehicle that requires maintenance, so if it is not kept in a good order, it will cease to function. The key concept that Fuller develops, as an ecological principle as well as a design criterion, is his notion of "ephemeralization," or "the doing of ever more with ever less, per given resource units of pounds, time, and living in ever-increasing numbers" (Fuller 26).

Spaceship Earth today is a worldview encouraging everyone on earth to act as a harmonious crew working toward the greater good. The concept of the earth with its finite resources had entered the Planetarium, too: the short science fiction story by Isaac Asimov entitled The Last Question was first adapted for the Abrams Planetarium at Michigan State University in 1966, featuring the voice of Leonard Nimoy, who is known for playing Spock in the Star Trek franchise, a character he portrayed in television and film for almost fifty years (Asimov 1980).

Then, in 1969, the literary piece was further played in the Strasenburgh Planetarium in Rochester, New York, under the direction of Ian C. McLennan and in the early 1970s, the piece was brought to life again for the Edmonton Space Sciences Centre in Edmonton, Alberta, under the direction of John Hault. The piece was then subsequently played, as well, in 1973, at the Fels Planetarium of the Franklin Institute in Philadelphia, in 1974, at the Neag Planetarium of the Reading School District in Pennsylvania and the Buhl Planetarium in Pittsburgh; in 1978, at the Vanderbilt Planetarium in Centerport New York read by singer-songwriter and Long Island resident Harry Chapin; in 1980 and 1989, at the Hansen Planetarium in Salt Lake City, Utah; in the early 1980s, Morrison Planetarium in San Francisco, California. Currently, in 2017, the adaptation that combined visual elements digitized from the original glass slides, Nimoy's recorded voiceover, and today's advanced planetarium technology was revived at the Gates Planetarium in Denver, Colorado. "Science has finally caught up with science fiction” (Boxer 2000) - a quote widely reprinted within Planetarium Hayden's own promotional materials (Quoted from McConville 216). The fictional supercomputer Multivac, appearing in the science fiction story by American writer Isaac Asimov, displays the message "Insufficient data for meaningful answer" as an error message, when asked by two scientists if humanity can reverse the increase of entropy which threatens with a possible cosmic disaster, bringing all life to an end.

Today, in the Anthropocene epoch, we seem to do the same gesture through the technology that is available to us by collecting, retrieving, analyzing information stored on our computers. The concept - that all information could be contained on computer(s) and 
accessed from a domestic terminal - constitutes in these science fiction literary pieces an early reference to the Internet as database and network, Big Data as information and all other technological advances that bring unimagined new possibilities into our realities. Computers have often been used as fictional objects in literature, movies, and in other forms of media, where they tend to be represented considerably more sophisticated than anything yet devised in the real world. In the science fiction story by Isaac Asimov entitled The Last Question, the computer named Multivac is growing ever more enormous with each section of the story and develops a certain relationship with humanity through the courses of seven historic settings, beginning in 2061, on the day that Earth becomes a planetary civilization. In each of the first six scenes, a different character presents the computer with the same question; namely, how the threat to human existence posed by the death of universe can be averted. The story jumps forward in time into later eras of human and scientific development. Again, in each of these eras someone decides to ask the ultimate "last question" regarding the reversal and decrease of entropy. Each time, in each new era, Multivac's descendant is asked this question and finds itself unable to solve the problem. The story continues through many iterations of computer technology, each one more powerful and ethereal than the previous. Each of these computers is asked the question, and each of them returns the same response until finally the universe dies. At that point, Multivac's final successor, the Cosmic AC, which exists entirely in hyperspace, has collected all the data it could, and so asks the question to itself. As the universe died, Cosmic AC drew all of humanity into hyperspace, to preserve them until it could finally answer The Last Question. Ultimately, Cosmic AC did decipher the answer, announcing "Let there be light!" ascending to the state of the God of the Old Testament or the Big Bang explosion or the moment of the invention of electricity or solar panel energy...so the saga/history can begin anew... From 1966 until 1980, 'The Last Question' was constantly asked in the Planetarium setting... Is it possible that the reason that Multivac doesn't find an answer for solving Entropy is what happened before he was even invented? The invention of electricity and the apparition of the very first projection based Planetarium marked the anthropocenic moment in astronomy... Or is it about these very moments that are not present in his complex calculations? What kind of data have we gathered until now? And by what means? What kind of relationship has humanity developed with the computer? Can we reverse the increase of entropy which is threatening with a possible cosmic disaster, bringing all life to an end? What can we find out if we question the Anthropocene condition through the Planetarium medium? Would the Planetarium embrace the science of the earth as it did with the sky?

These questions seem to resurrect from the science fiction literary piece into our contemporaneity, inherently following us in the next chapters striving to get answered, but also to maintain us alert and critical towards the problematic they portray. 


\section{Visualizing the Anthropocene as Interacting Spheres}

Entropy, as fundamental function of the state of being, became our main concern under the name of Anthropocene, an epoch that has not any agreed exact start-date and location, but only proposals, based on diverse evidences. Too big and complex to grasp, the Anthropocene epoch seems to be easier to analyze if it is continuously divided into anthropocenic moments analyzed for being the starting points in generating a substantial degradation in all the four major Earth subsystems: land, water, living things, and air. Earth scientists call these four subsystems "spheres". Specifically, they are the "lithosphere" (land), "bydrosphere" (water), "biosphere" (living things), and "atmosphere" (air). Each of these four spheres can be further divided into sub-spheres resembling a puzzle ball, an artefact that consists of a number of intricately carved concentric hollow spheres carved from a single solid block that fit within one another in a way that looks impossible (Schaumberg 2017). Earth science traditionally viewed and taught these spheres isolated from one another through subjects like geology, biology, chemistry, weather. However, a new perspective on Earth science was needed as in the real world, almost nothing occurs in isolation - the earth is a system of interconnected parts always interacting with and affecting each other. Building on the recognition that earth is driven by the diverse interactions between energy, matter and organisms a new science was founded: Earth System Science (ESS). As an integrative field, it evolved in the 1980s, particularly at NASA, where a committee called the Earth System Science Committee was formed in 1983. The earliest reports of NASA's ESSC, Earth System Science: Overview (1986), and the book-length Earth System Science: A Closer View (1988), constitute a major landmark in the formal development of Earth system science (Mooney, H. A., et al. 2013). Early works discussing Earth system science, like these NASA reports, generally emphasized the increasing human impacts on the Earth system as a primary driver for the need of greater integration among the life and geo-sciences, making the origins of Earth system science parallel to the beginnings of global change studies and programs. This new science had as precursors the above mentioned earth related movements too, along with other continuous discoveries and since its establishment it has produced innovative concepts and frameworks central to the global-change discourse, including the Anthropocene (Steffen, Will, et al. 2020). Earth system science (ESS) is the study of cycles and interactions among the various components, or spheres, of Earth along with the human generated impact. As earlier mentioned, these spheres are not static, but constantly changing, configuring a dynamic Earth: the atmosphere does not produce the same weather every day; we experience the wind, rain, or sunshine differently every day; living things such as animals are also born, while older ones die; seismic movements cause changes in the earth's crust, and molten magma from deep down the Earth can be spewed in the form of lava during volcanic eruptions. Earth's spheres work together as a complex system intersecting and affecting each other. The processes that 
move matter and energy from one sphere to another are defined by Earth System scientists as "sphere interactions". Also, as the four spheres are interconnected, human impact on one sphere will potentially affect other spheres: the release of plastic pollution into the oceans (hydrosphere) will impact the marine life (biosphere); the production and release of CFCs into the atmosphere will affect the impact of UV radiation on the biosphere, etc. Moving forward, the grand challenge for ESS is to achieve a deep integration of biophysical processes and human dynamics to build a truly unified understanding of the Earth's spheres. The evolution of these impacts that led to the Anthropocene epoch seems to envelop the Spheres of the earth with a new spherical layer - the Anthroposphere, also referred to as Technosphere. Professor Zalasiewicz explains: "The technosphere is all of the structures that humans have constructed to keep them alive, in very large numbers now, on the planet: houses, factories, farms, mines, roads, airports and shipping ports, computer systems, together with its discarded waste" (Zalasiewicz 11). Homo faber or the manifestations of humanity's intelligence, a concept referred by Henri Bergson in Creative Evolution (1907), defines intelligence, in its original sense, as the "faculty to create artificial objects, in particular tools to make tools, and to indefinitely variate its makings".

Inspired by Henri Bergson's notion of Homo faber, Vladimir Verdansky devoted a great deal to the understanding of the intellectual and technological developments of human civilization in the Biosphere. To explain this new anthropogenic interference with biogeochemical processes and Earth history, Verdansky introduced an additional sphere, the Noosphere, to identify a new stage of the biosphere's evolution in which the human mind, through scientific research and engineering development, becomes the main driving force for global environmental change in future-Earth history (Verdansky 1930). Every added Sphere generates complex interactions as the puzzle ball consisting of nested spheres can rotate freely inside each other, showing always new configurations. The Earth's spheres which already have their own dynamic are constantly affected by the constant changes generated in the Noosphere that modifies the Anthroposphere and vice versa. The interacting spheres model the kind of interdisciplinarity that the epoch of the Anthropocene requires. As we seem puzzled in front of these nested Spheres, that can be analyzed in different periods and through processes that are neither homogeneous nor linear, we find ourselves navigating inbetween sub-spheres realizing that Anthropocene is not only a current geological epoch per se but can also be measured as a reflexive state of being as stated by the idea of the Noosphere: human reason and the scientific thought have created and will continue to create the next evolutionary geological layer.

Thus, the spherical encounters of the Anthropocene firstly refer to the nested spheres as a dynamic force in constant motion, the circulation of elements across the entangled geospheres and their new configurations and secondly to their intermedial and multimodal representation through the Planetariums which function as a cultural interface for all these 
ecological expressions. We have to use an environment to tell a story about an environment. That is, to create a situation where the experience of the spatial media creates a new correspondence with the space being represented. The story of these interacting spheres as complex environments seems to fit perfectly onto the spatialized screen and listened closely through the surround sound environment of the Planetarium. These major spheres in motion rotate in space and time accordingly to re-observe their evolution, the newly resulted connections and the current state of representation to which they lead.

\section{Double Vision: the Dome as Sky and Earth}

The big change in how planetariums are able to envisage the cosmos has come through the rapid development of computer technology and the ability to store and project vastly greater quantities of information. Improvements in projection technology evolved quickly in the 1980s, altering the nature of the planetarium show. Maryland Science Center was the first to use the All-sky system of six slide projectors, each with customized wide-angle lens and set up to project across 60-degree triangular segments of the dome. The images projected onto the dome were not necessarily astronomical, but could be taken from art or weather conditions, so that the dome could be used to create an illusion of being elsewhere. Images of architecture could be projected using All-sky system, creating for instance the impression of being under the dome of St. Peter's Basilica, or in the starry hall of the Alhambra. The Digistar, the first digital planetarium projector, invented by the digital graphics firm Evans \& Sutherland and first installed at the planetarium in Richmond, Virginia, in 1983, projected any sequence of images produced by the computer software onto the dome through a single fish-eye lens. Planet earth got invaded by a smaller breed of artificial planets, within which are displayed the wonders of the cosmos - projected planets-within-planets, constructed in three dimensions (Firebrace 192). The technological requirements for high quality geoscientific visualizations have taken about a decade longer to mature than they have for the astronomical features of digital planetariums, and they still have to catch up, but Earth System Science (ESS) is finally adopted by the Planetarium as a complex new program: with the release of Uniview 1.0 in 2005 from SCISS and initial releases of the Worldviewer software from Elumenati in 2009-2011, the capacity to show earth at high levels of detail became available to digital planetariums. After a handful of pilot tests in 2007, Denver Museum of Nature and Science (DMNS) began offering a regular bi-monthly live evening lecture series titled Digital Earth. Because gross earth surface features were easily seen in landsat imagery available from remote WMS (web mapping service) servers, many of the initial Digital Earth topics focused on geography, geology, and natural history. However, given the expertise and interests of the presenters, there were an increasing number of talks on Earth Systems and 
global environmental change. Per absurdum, the digital technology in the planetarium thus reconnects our ontological link to Nature through satellite images showcasing not only re-created imaginaries but also direct data about our climate conditions: the explosion of information being returned from satellites and other remote sensing platforms has increased the availability of geospatial data highlighting any number of Earth phenomena (e.g., see Overpeck et al. 2011 for climate data). Nasa Landsat offers the longest continuous global record of the Earth's surface: for over 40 years, the Landsat program has collected spectral information from Earth's surface, creating a historical archive in quality, detail, coverage, and length. "It was the granddaddy of them all, as far as starting the trend of repetitive, calibrated observations of the Earth at a spatial resolution where one can detect man's interaction with the environment," says Dr. Darrel Williams, the Landsat 7 Project scientist (NASA website). Collected data during 50 years of space missions by manifold sensing media such as robotic probes, telescopes, spacecrafts, sensors cris-crossing the face of the globe are translated into "accurate imagery" to create $3 \mathrm{D}$ worlds that live in the computer and can be shown on the planetarium screen (Trakinski, Business Insider 2020).

In his book, Software Takes Command, Lev Manovich explains that Google Earth is an example of a new genre of media that "does not have any fixed finite boundaries" (Manovich 36). In the book review, Yanni Alexander Loukissas writes: "In Google Earth, you can never click on the same river twice. The software application is not a fixed image of the earth, but rather a worldwide network of media in flux" (Loukissas 107). Using OpenSpace, an open-source software platform that the Hayden planetarium developed with NASA, the filmmakers can convert raw mission data into detailed 3D visualizations, building intricate photo-mosaic fly-throughs even in real time. It is basically a very advanced version of Google Earth, but for the universe (Pasternack 2020). Anthropocene in the Planetarium is addressed not only globally but at a planetary level.

These methods have also been approached since the view of the night sky from the surface of the earth, which had been the traditional way of pursuing astronomy. As a consequence, the sky has become increasingly obscured: "it is the first time in human history that we have lost the direct contact with the night sky. One third of humanity cannot see the Milky Way," said Fabio Falchi, physicist, working on light pollution for more than 25 years. In June 10, 2016, a joint study entitled The new world atlas of artificial night sky brightness was published in the open access journal Science Advances. The project was led by principal investigator Fabio Falchi with Light Pollution Science and Technology Institute (ISTIL, Italy), and with additional expertise from Duriscoe, the National Oceanic and Atmospheric Administration (NOAA, USA), Deutsches GeoForschungsZentrum (GFZ, Germany), and University of Haifa (Israel). They found that about $80 \%$ of the world's population and more than $99 \%$ of the U.S. and European populations live under light-polluted skies. Due to light pollution, the Milky Way is not visible to more than one-third of humanity, including $60 \%$ of 
Europeans and nearly $80 \%$ of North Americans. Moreover, 23\% of the world's land surfaces between $75^{\circ} \mathrm{N}$ and $60^{\circ} \mathrm{S}, 88 \%$ of Europe, and almost half of the United States experience light-polluted nights (Falchi, Fabio, et al., 2016). Their study on light pollution was painstakingly produced over the course of more than 10 years from satellite data and verified by more than 30,000 on-the-ground measurements. As illumination from streetlamps and other artificial illuminations reaches into the night sky, it is reflected off moister droplets in the atmosphere, leading to a 'sky glow'. This light pollution, which would reduce if for some reason all the electric lights in the cities and on highways were switched off, is a sign of a condition that has steadily evolved since the days of Oskar von Miller (Firebrace 176-7).

At the same time, as we will later see, from an ecocritical perspective, this paradox contradicts itself in the moments when the continuous technological advancements and city pollution made the Planetarium space to signify a safe and quiet place where re-connection with Nature and our cosmic consciousness gets possible: “...Planetaria allowed visitors to escape from the light-polluted confines of an urbanized landscape and to re-experience the splendors of the stars as they had been known in preindustrial times” (Marché 146); and the "planetarium's strikingly realistic depiction of the night sky offered a respite for city dwellers from the growing impact of light pollution associated with modern urbanization and industrialization" (Marché 15). If, at the creation of the Planetarium, the real sky experience was replaced by a media simulation, today Nature is available to us only through this representational machinery conveying the most direct images about the Anthropocene condition.

\section{From Telescope to Kaleidoscope}

For the first time in history, the physical survival of the human race depends on a radical change of the human heart - Erich Fromm

While approaches to art and science have long been separated in history, there is a broad consensus that there is something to be gained from encouraging artists and scientists to collaborate (Rodder 2016). Using a multidisciplinary approach to combat issues as expansive as climate change has the ability to provide a more diverse range of viewpoints, developing solutions that may incorporate aspects of multiple fields. By combining the arts and humanities with environmental sciences, new ways of thinking are sparked (Hayley 2019). How are the diverse scientific media appropriated by artists and put into intermedial relations to communicate topics about Anthropocene in various audio-visual productions? The lenses of the telescopes and satellites extend our scientific vision far away into the Universe to unfold its mysteries while the Kaleidoscopic lenses - representing creativity and playfulness - apply a hallucinatory and colorful filter to our vision to express our dreams 
and imagination through a multifaceted reality. The scientific and imaginative visions fused into interconnected, parallel universes can challenge our mundane notions opening up new causal connections, creating exciting discoveries, all under the notion of Anthropocene. As we will further see, the constant scientific discoveries and technological advancements are altering the nature of the Planetarium programming towards humanities and diverse medial expressions, including artistic practices exactly to foster a broader understanding of environmental complexities. By translating scientific data visualizations from pure information into narrative experiences, these shows invoke a planetary consciousness and social responsibility. Immersion as a technique builds an embodied connection with the projected environments: equipped with SCISS' Uniview, Denver Museum of Nature and Science worked with Kenji Williams on Bella Gaia in 2007, a program that combined live music and earth visuals (Yu et al. 2009). Bella Gaia is a live concert that mixes music, dance and satellite imagery of earth, time lapses, cultural heritage footage, NASA data visualizations to tell a cosmic story. By visualizing our effects on the biosphere the project is illuminating the relationship between civilization and nature as we enter the Anthropogenic Age. Bella Gaia is a new story for our time, delivered in the language and technology of the future, according to the information provided by the project creators on their website. Satellite photography along with surveillance cameras are imaging practices from which humans are absent. These practices are largely defined by Joanna Zylinska in her book entitled Nonbuman Photography (Zylinska 2017). As satellite imagery is decoupled from human agency and human vision the resulted photographic images are domesticated and objective. As artists add to these images music, performative events they add their subjective filter. By presenting these objective and subjective perspectives to large audiences the Planetarium is transforming these projects into intersubjective topics. Here we arrived at an interesting subject, a twofold debate regarding the Anthropocene that lurks between the human hubris and human guilt. While the approach of grand scales achieved through the cosmic zoom works well in understanding the interactions that maintain our existence and observe disharmonies, the same scale placed to humans seems to provoke further debates. While the term Anthropocene implies that humanity, humans in general, are responsible for bringing about the new geological age and that "humans as species are perceived in its finitude" (Di Blasi 2020), we know that behind each statistic there is a person - a parent, a spouse, a sibling, a neighbor, a friend. Every person is valued, each life is worthy. In a workshop entitled Guilt in the Anthropocene organized by the University of Vienna, Luca Di Blasi in his abstract entitled Guilt and Finitude writes:

Pessimist interpretations of the Anthropocene and the climate change confront us with the very possibility that we might have already crossed the point of no return and that the end of (large parts of) humanity can no longer be halted. By changing the scale from the individual to the species, mediating a collective human 
guilt, including the possibility of a self-inflicted finitude of the human species can lead to an acknowledgement of an "existential human guilt" (Di Blasi 2020).

These sentiments come in contrast to the hubris of the Anthropocene, as expressed below:

At the apex of his insanity, Man has even proclaimed himself a "geological force," going so far as to give the name of his species to a phase of the life of the planet: he's taken to speaking of an "anthropocene." He observes the rarefaction of life on earth from space. He has the hubris to claim, paternally, to be "protecting the environment," which certainly never asked for anything of the sort... But who is this Man?" (To Our Friends 32-33).

The Planetarium addresses this question with a third attitude: in the 1930s, at a moment of existential crisis comparable to today's, Husserl likewise sought to reorient science around shared human experiences and common human needs. "To save society, science should embrace ethical projects" writes Deborah R. Coen in her book review entitled Knowledge in the Anthropocene, putting thus the emphasis on a new type of science proposed by Jürgen Renn in his book The Evolution of Knowledg. He argues here that "modern science, rather than striving to be value-free, should embrace ethical projects of the sort usually associated with religion" (Coen 256). In his book The Overview Effect: Space Exploration and Human Evolution, Frank White (1998) relates to space travel experiences as "spiritual technologies" that "maintain a link to the spiritual experience of the universe, generating a relationship with God, an ultimate oneness, 'the Universe,' or 'System of Systems”' (Quoted from McConville 78). The Planetarium doesn't address the human guilt or hubris, but common human ethical projects. For example, the movie Fragile Planet (2008) gives the audience an astronaut's view of the earth, highlighting unique regions that we may lose. The movie may trigger the Overview Effect, the affective response reported by astronauts who viewed earth from the orbit (White 1998), but this time the effect can be perceived at a large scale by the whole Planetarium audience again and again, configuring a sort of a generational reenactment. White describes this experience as a "cognitive shift in awareness with a new understanding of humanity and our place in the universe" (Quoted from McConville 303). In the Planetarium, the intersubjectivity is our fragile home, our earth, we as human species and our existence that depends on the well-functioning of the earth itself. Every time the quest of a planetarium movie is to search for habitats that might host extraterrestrial life, we find that there is no other Life found anywhere in the cosmos... There is only One Earth that is counting to one and no more (Schneider 2008). The importance of one earth, considered as a totality, emerged in tandem with early space travel and rising concerns about an imminent nuclear apocalypse (Joshua 133). As external architectural forms, the planetariums are naturally reminiscent of planets and other celestial bodies, but inside on 
the curved screen of the dome a new projection chapter begins by bringing the apollonian reverse gaze sensation to the wide audiences. Looking up, but seeming to look down to earth without changing any seat position, is reflected best in a new catch-phrase from California Academy of Science's Morrison Planetarium: "We put the earth in the planetarium."

Anthropocene in the Planetarium is always presented through the diverse Sphere interactions in a comprehensible way. The significance of a topic in the fulldome planetarium can be measured by how many pre-recorded programs have been developed on it. Global change may have enjoyed priority in the adaptation by the planetarium world since it is one of the most rapidly evolving geoscientific phenomena and is even of political significance (Yu et al. 2014). The Planetariums seem to show our world through contrasts: while the cinematic show Dynamic Earth (2012) explores the inner workings of earth's great life support systems using visualizations based on satellite monitoring data and advanced supercomputer simulations, the cutting-edge production follows a trail of energy that flows from the sun into the interlocking systems that shape our climate: the atmosphere, oceans, and the biosphere. To have the Overview Effect (White 1998) can be life changing, but what is on the other side of the coin? Space debris, also known as space junk, space pollution is made up from the satellites that humans launched into orbit and have stopped working, and some have crashed into each other and broken into tiny pieces. All those bits and pieces, and the old "dead" satellites, have become "space junk" that whizzes around the earth at superfast speeds. The planetarium film Space Junk (2019) screened at the Eos Planetarium Theater, traces the history of human-launched satellites and the origins of space junk, including some of the famous satellite collisions that have added to the growing ring of debris that orbits 22,000 miles above our planet. Along with the immersive sensory experience, the film also introduces Don Kessler the pioneering NASA researcher known as "The Father of Space Junk." To shift the basis of the technosphere's horizontal emphasis toward the orbital layer of satellites and debris that constitutes the technosphere's vertical expansion - the vertical expansion through an orbital layer of satellites (Gärdebo 2016). The repetitiveness of the Anthropocene as main subject in these productions is always grasping to keep-up with the speed and levels of endangered regions, species and occurring phenomena. Mission Earth (2019) sets the message that it is time to act upon the situation now, by presenting the interaction of the forces of nature out of balance, while Our Living Climate (2009) tells about the history of climate change on Earth at large. To describe the increasingly unpredictable world, the German sociologist Ulrich Beck introduces the term 'reflexive modernization' and argues that scientific and technical advances bring unquestionable benefits, but they also generate new uncertainties and failures, with the result that doubt continually undermines knowledge, and bring unforeseen consequences (Beck 2003). As an antidote for these uncertainties and the partiality of knowledge, but also for human claims of omniscience, come the 'technologies of humility' theorized by Sheila Jasanoff: "If we keep 
the negative distributive impacts caused by new and emerging technologies under control, and consider lower-impact alternatives as needed, then we can use technology not to harm or destroy the world but to make it a truly better place" (Jasanoff 2007). Indeed, what the cosmos has to teach us is that our knowledge is limited and depends on new discoveries but also that our way of perceiving is delayed - seeing the phenomenon of a falling star shining now might have occurred 100 years ago in space. Cosmic images reach our eyes with a considerable delay. So does the Anthropocene - what we perceive actually started long time ago. To counteract the delay, each Planetarium production seem to hold a red flag, to make the red danger light beep and pulsate, re-enacting the fragility of the earth to each generation again and again: Super Salvador (2019) depicts a youthful and charismatic super-hero who invites children on a mission to help solve environmental problems in a very entertaining and clever manner: "If you also want to be a superhero, join us on this adventure and let's save our planet!" Planetarium productions addressing Anthropocene to children had grown into a separate genre and their main message is the use of renewable energy sources and recycling. Instead of turning these productions into green jeremiads of the Anthropocene era to dramatize the crisis, the experience is placed on shared values and new methods of possible sustainable practices.

The immersive space of the Planetarium thus acts as a creative environment, a platform fostering collaboration, communication, empathy, creative agency, and connectivity through multiple disciplines and working methods. The level of collaboration that these shows imply is impressive. Just to name an example, Dynamic Earth (2012) show is the result of a twoyear long collaboration between Spitz Creative Media, the Advanced Visualization Lab at the National Center for Supercomputing Applications (NCSA) at the University of Illinois, NASA's Scientific Visualization Studio, and Thomas Lucas Productions, Inc. produced in association with the Denver Museum of Nature \& Science and NASA Earth Science.

The Anthropocene represented in the Planetarium reminds us of the necessity to think at more-than-human scales: considering deep time and vast space as well, reflecting on complex processes, the Anthroposphere is addressed not only globally but at a cosmic level. The common element employed in all Planetariums is the orders of magnitude, a class in a system of classification determined by size, typically in powers of ten. This is achieved by zooming from a micro to a macro scale, or from a normal view to macro and then back into the micro.

Thus, the Anthropocene in the Planetarium is seen between the overview and detail at all scales. A pixel is generally thought of as the smallest single component of a digital image, which today arrives at the highest levels of digital values: gigapixel, terapixel, gigapan. High resolution maps about earth and cosmos are available today through dedicated software. Through zooming out,e becomes a dot on the screen, through zooming in, the invisible is made visible. 


\section{Interactive ArtSpheres}

While the majority of the Planetarium shows focus on narrating the scientific and natural descriptions of the Anthropocene, the interactive and haptic projects seem to enact inner spaces of psychological and social processes through forgotten embodied perceptions. These artists seem to readdress the globe, global and globalized through sphairopoiia, the Greek art of sphere-making (Evans 262) by extensive use of the material artifact and the environment of the sphere.

The question of reversing Entropy addressed to Multivac resurrects today through a myriad of pulsating light of leds that illuminate our contemporaneity with algorithmic radiance through state-of-art spheres connected to the computer that are becoming complex expressive surfaces: Entropia (2014) is a live performance that is based on an audio reactive geodesic led sphere that keeps a constant interaction with the generative visual projections on the inner surface of the Satosphère, an $18 \mathrm{~m}$ immersive theater dedicated to immersive art. The sound artist stands inside the led sphere, while the other team members perform real time pixels mapping and visuals. The audience is placed in the interstice created by the duality of physical light of the geodesic sphere and external walls projection, filled by cutting-edge sonic material visuals made by using real time, 3D programming. A spherewithin-a sphere, the project aims to create an immersive scape made of interconnected layers of various media using a multi-modal experience by mixing sound, architecture, design, physical light, and $360^{\circ}$ visuals. The geodesic led structure is used to pay tribute to the ideas of Buckminster Fuller whose mission was the implementation of a sustainable development in a world with finite resources. Entropia, the title of the performance, states that any real transformation occurs with inevitable increase in global disorder; disorder measured by entropy, a law that involves several questionings for our own civilization progress and our duty to necessarily think of a sustainable development. But the question is how to achieve this sustainability? This is what the two scientists asked Multivac in the science fiction story too. Another led installation resembling a geodesic dome entitled Konstellation (2019) wishes to question our relationships with the worlds around us. In a constantly hyperstimulating and changing city we often feel disconnected from Nature. The artist proposes to bring back the stars through computer programmed rhythmically pulsating led patterns that are to be viewed from the inside outwards toward the stars. The creator's statement is to virtually recreate our lost paradise by stimulating interrogation while arousing wonder. The Disenchantment of the world, a phrase borrowed from the poet Schiller, as Charles Taylor and later Max Weber and also Alkis Kontos theorized it, is a world driven by individualism, the overvaluation of rationality, and the political implications in a world of individualism and instrumental reason. However, through 'disenchantment' (Entzauberung), Weber had in mind the distancing from the immediate experience of nature - and, indeed, the 
experience of the sacred in nature that had predominated in the medieval mind - through the emergence of the modern scientific viewpoint, and the increasing rationalization and bureaucratization of society enabled by the technological and economic advances of the age, which together created a sense of alienation of the individual, from the natural environment and the social other (Trubshaw 2017). Kontos asserts that it is rationality that is the underlying drive of disenchantment. Rationalization seeks to empty the world of any sense of magic, mystery, and thereby any meaning. "Disenchantment" literally means "de-magication." It is rationalization that deduces our human experience so that emotion and soul, which is a central characteristic of humanity, is either irrelevant or absent. With rationality, humans are left devoid and anaesthetized (Shah 2020). The Enchanted sense of the World was defined as one filled with meaning and integration. People were in a constant dialogue with their communities and nature when living in a symbiotic relationship with their environment. The cosmos and natural world was alive in the human eye: the sky provided characters, mythologies, storytelling experiences, and the sense of the sublime. However, the mechanization and rationalization of the Sky through science and technology, leading to the telescopic and satellite vision, brought an intermedial re-enchantment, regaining our sense of wonder and awe through the Planetarium by artistic practices, or as it is mentioned here, the kaleidoscopic vision.

Bruno Latour (1990) addresses the importance of visual inscriptions and their enabling technologies within this transformation, connecting visualization to cognition through what he calls "thinking with the eyes and hands" (Latour quoted by McConville 47). The sky gets translated by artists to our senses visually and auditory, but can we really touch the skyline? The public art installation entitled $O H !$ (2018) invites the audience to touch the skyline with their hands. $O H$ ! is a 1:60 scale model of the Science World, the iconic building of Vancouver which is positioned on the edge of False Creek river and its unique geodesic shape can be seen from almost every vantage point in the city. The miniature replica is covered with 240 LED lights and 240 sensors that 'feel' the movement of people's hands as they touch the model. The sensor data is sent to the actual lights on Science World via wireless cellular connection and changes the patterns of light on the landmark structure in real time. OH!'s software was developed to include six different animations of varying colors and patterns, all of which can easily be switched on-site through a custom app on a smart device. The Telus World of Science Edmonton in Vancouver was built for Expo 86 and was inspired by Buckminster Fuller's geodesic dome. However, the installation $O H$ ! seems to materialize Fuller's unrealized Geoscope. In 1962 he proposed to create a 200-foot-diameter $(61 \mathrm{~m})$ globe, which would be covered in colored lights so that it could function as a large spherical display. It was envisioned that the Geoscope would be connected to computers which would allow it to display both historical and current data, and enable people to visualize large scale patterns around the globe (Fuller 1962). Many of Fuller's ideas for the 
functions of the Geoscope are now being realized by virtual globes called Science On a Sphere. Fuller paraphrased from Critical Path says: "We also need to construct many Geoscopes, which are large, see-through spheres shaped and oriented like the planet earth. By standing inside, you can view the stars exactly as they appear to anyone standing at any point on earth. Computers for each Geoscope will "store all relevant inventories of world data arranged chronologically, in the order and spacing of discovery, as they have occurred throughout all known history." Time-lapse images projected onto the Geoscope will display in a matter of minutes all sorts of global, long-term trends, everything from continental drift to human migration to use of transportation. With the Geoscope humanity would be able to recognize formerly invisible patterns and thereby to forecast and plan in vastly greater magnitude than heretofore" (Fuller 1981). "Working together at the intersection of art and science to create experiences that evoke a sense of wonder and engage the public at this scale is super exciting. The goal was to create a space for social interaction and we feel that $O H$ ! does just this," Tom Cummins, director of exhibitions at Science World, says (Chan 2017)."The future is what you make of it! We cannot foresee the future, but we can shape it" remarks Martin Kutsch, one of the main members of the project that functions as a metaphor for a predicting machine, displaying a set of questions, pieces of information, and visions of the near future of our society: The Future Room (2017/2020) is a participatory installation based on a speech recognition system. It is a virtual knowledge space that shows us imagined futures in which visitors can interact via a voice recognition system. The work takes the form of fourteen information clouds visualized in an immersive dome environment. Visitors are invited to choose one of the themes by speaking into a microphone. This sets off the screening corresponding to their choice: Artificial Intelligence, Education, Energy, Evolution, Finance, Genome Editing/CripsR, Information, Quantum Physics, Migration, Politics, Religion, Urbanisation, or Work. Today Asimov's giant centralized computer is part of our everyday reality as we are asking questions from Siri, Alexa, Cortana, Bixby, Viv, Alice, Google Assistant, and we increasingly rely on anthropomorphised artificial intelligence - powered voice assistants in our homes or in our hands for weather, news, homework help and such. The Future Room is both utopia and dystopia. According to the project's motto, the Future Room provides thought and visions for the future. Encapsulated in a cloud of information, the future will only be visible where we strive to imagine it. From abstract generative animations to politically relevant statements, the animations treat the dome as an intermediary membrane between the inside and the outside, a thin layer between disenchantment and re-enchantment. The project allows for multiple, non-hierarchical entry and exit points in data representation and interpretation. It is very true that the method of our time is to use not a single but multiple models for exploration. 


\section{Conclusions}

Immersing viewers inside large panoramic image "bubbles", or spherical images rendered completely digitally called as fulldome masters, means immersing people in places from the past, present, or future. A suspension in another space and time happens. Foucault uses the term heterotopia to describe spaces that have several layers of meaning or relationships to other places than it immediately meets the eye and describes certain cultural, institutional and discursive spaces that are somehow 'other': disturbing, intense, incompatible, contradictory, or transforming (Foucault 20). New Media scholar Hye Jean Chung applies the concept of heterotopia to describe the multiple superimposed layers of spatiality and temporality observed in highly digitized audiovisual media. She is expanding Michel Foucault's concept of heterotopic spaces to encompass the digitally composited environments in contemporary cinema, introducing the critical concept and methodology: "media heterotopia"(Chung 1737). In her book, she demonstrates the use of "media heterotopia" as a mode of perception that recognizes and describes new hybrid forms, that is, digital composites of multiple layers that contain material residues of globally dispersed film production. It examines industry practices that take place in the workflow of global production pipelines in a digital era: "Because digital compositing is an integral stage in film production that achieves the technical and aesthetic merging of multiple digital layers, assets, and environments, the critical appropriation of this term is apposite to analyze the site-specific conditions of contemporary film production and the effects they produce" (Chung 3). In our case, the screen architectonics imitating the ancient sky coupled with state-of-art imagery generates not only a representation of space, but spatial experiences as well, so my aim here is further contextualizing the "media heterotopia" concept as "liminoid media heterotopia": in 1974, Victor Turner coined the term liminoid to refer to experiences that have characteristics of liminal experiences but are optional and do not involve a resolution of a personal crisis. Unlike liminal events, liminoid experiences are conditional and do not result in a change of status, but merely serve as transitional moments in time. The liminal is part of society, an aspect of social or religious rites, while the liminoid is a break from society, part of "play" or "playing" (Turner 85). With industrialization and the emergence of leisure as an acceptable form of play separate from work, liminoid experiences have become much more common than liminal rites. In these modern societies, rites are diminished and 'forged the concept of "liminoid" rituals for analogous but secular phenomena' such as attending concerts and other liminoid experiences. Edith Turner, Victor Turner's wife, further develops the idea and uses the term "Communitas" referring to an unstructured state in which all members of a community are equal, sharing a common experience, usually through a rite of passage. The transient personal experience of togetherness which occurs during events generates existential or spontaneous communitas (Edith Turner 2011). Indeed, in the Planetarium 
and ArtSpheres, the limit of our knowledge and our existence becomes questioned through multiple types and layers of media in a 'playful' manner. However, this is my personal interpretation of possible spectatorial experience. To attest this, audience research should be done through questionnaires or video interviews.

Thus, experiencing the Anthroposphere in the Planetarium functions in-between the 'liminoid' - as we experience representations of the Anthropocene - and 'liminal' - while in reality we are in the Anthropocene already.

Planetariums and ArtSpheres, with their spherical screen imitating the sky and the earth are exploring at a very intimate level the role of human imagination in shaping the Anthropocene. Through the integration of both artistic animations and scientific visualization, participants are taken to the heart of Earth's regenerative systems and invited to re-imagine humanity's relationship to our home planet. Strategies for sustainable transformations are needed, as we have to fundamentally rethink our existence. By evoking the imagination beyond our daily experiences, these programs reveal complex interpretations of an interconnected and re-enchanted world. Our "post-media condition" (Weibel 2006) in which "no single medium is dominant any longer; instead, all of the different media influence and determine each other" mimics the anthropogenic mechanism: all life elements are important; we cannot reduce Anthropocene to one element but to several influences that continuously determine each other. The analyzed Planetarium and immersive dome art projects seem to function as living spherical organisms existing in various systems of balanced symbiotic relationships creating all together art ecosystems or new constellations that address the anthropogenic condition with multiple and diverse meanings in which personal, social-cultural, historical, and aesthetic dimensions coexist. The projects create a dialogue between the academy, industry, and the public sphere; between theoretical and applied knowledge to re-question contemporary topics such as environmental issues, recycling, new materials, regenerative practices, and the role of computers, data sensors, data visualizations. These immersive spaces are perceived by the public as creative environments fostering collaboration, communication, empathy, creative agency, and imaginative play generating contact, connectivity, culture and a platform for social innovation in science and art projects. The Planetarium cinematic shows shape our knowledge and consciousness by learning and understanding how the sphere interactions manifest and then how we humans further alter them. ArtSpheres address the Anthropocene at the intersection of technology and physical sensation surveying our emotions. Planetariums and ArtSpheres act as spaces for spherical encounters of the Anthropocene represented through immersive cinematic narratives and interactive art installations all connected to and fed by "the post-media computer, the universal machine" (Weibel 2006).

How do we feel the Anthroposphere? These projects hold a contrast between the telematics and the physical presence necessary to achieve the interactivity embedded in 
the artworks: while interactivity and the haptic objects require the physical presence of the participants, telematic art is the networked presence through technology. These ArtSpheres require the very earthly presence of the participants: their touch and wonder $(\mathrm{OH} !)$, their voice (Future Room), and their ancestral consciousness (Konstellation), to make them feel not only globalized citizens but cosmic entities. Displayed locally, Planetariums and ArtSpheres embrace our global concerns to transcend them. From the here and now we flow along within the infinite Universe where feeling bodiless we float but gravity like a magnet drags us home from the outer space to the inner reaches of our human heart. Our postures in relation to these Spherical encounters are ultimately metaphysical. Cybernetic serendipity became our medium to attend cosmic revelations in these Planetariums and ArtSpheres. For now we should let Multivac do his complex calculations and come back later to see what he came up with.

\section{Works Cited}

Asimov, Isaac. In Memory Yet Green: The Autobiography of Isaac Asimov. First Edition, Avon Books, 1980.

Atkins, Robert. ArtSpeak: A Guide to Contemporary Ideas, Movements, and Buzzwords, 1945 to the Present. Third, Abbeville Press, 2013.

Beck, Ulrich, et al. "The Theory of Reflexive Modernization." Theory, Culture \& Society, vol. 20, no. 2, 2003, pp. 1-33, doi:10.1177/0263276403020002001.

"Bella Gaia Beautiful Earth.” Bella Gaia - Beautiful Earth, 2019 Remedy Arts, www.bellagaia.com/ events.html. Accessed 16 June 2020.

Benjamin, Walter. Modern Classics One-Way Street and Other Writings (Penguin Modern Classics). Penguin Classic, 2009.

Boulding, Kenneth E. "The Economics of the Coming Spaceship Earth.” Resources for the Future, edited by H. Jarrett. Baltimore, Johns Hopkins University Press, 1966, pp. 3-14.

Business Insider India. "New, Detailed Pictures of Planets, Moons, and Comets Are Neither Photos nor Animations - They're Made Using Da." Business Insider, Times Syndication Service, 22 Jan. 2020, www.businessinsider.in/slideshows/miscellaneous/new-detailed-pictures-of-planets-moonsand-comets-are-neither-photos-nor-animations-theyre-made-using-data-from-50-years-of-nasamissions/slidelist/73503737.cms. Accessed 17 June 2020.

Chan, Kenneth. "You Can Now Use This Orb to Change the Lights at Science World (PHOTOS, VIDEO)." Venture Vancouver, Daily Hive, 11 Aug. 2017, dailyhive.com/vancouver/science-worldlight-control-art-installation-2017. Accessed 17 June 2020.

Chung, Hye Jean. Media Heterotopias: Digital Effects and Material Labor in Global Film Production. Illustrated, Duke University Press Books, 2018.

Coen, Deborah R. "Knowledge in the Anthropocene." Science, vol. 367, no. 6475, 2020, pp. 256, doi: 10.1126/science.aba1244. 
Committee, The Invisible, and Robert Hurley. To Our Friends (Semiotext(e) / Intervention Series). Illustrated, Semiotext(e), 2015.

DeMarrais, Elizabeth, and John Robb. “Art Makes Society: An Introductory Visual Essay.” World Art, vol. 3, no. 1, 2013, pp. 3-22, doi: 10.1080/21500894.2013.782334.

“Dynamic Earth - Fulldome Show.” Fulldome Database, 2012, www.fddb.org/fulldome-shows/ dynamic-earth. Accessed 16 May 2020.

Directorate, NASA Science Mission. "Earth Art Ebook.” Earth as Art NASA, National Aeronautics and Space Administration, 12 Nov. 2012, www.nasa.gov/connect/ebooks/earth_art_detail.html. Accessed 16 May 2020.

Elleström, Lars. Media Borders, Multimodality and Intermediality. Palgrave Macmillan, 2010.

Falchi, Fabio, et al. "The New World Atlas of Artificial Night Sky Brightness.” Science Advances, vol. 2, no. 6, 2016, doi: 10.1126/sciadv.1600377.

Firebrace, William. Star Theatre: The Story of the Planetarium. Illustrated, Reaktion Books, 2017.

Flusser, Vilém. “On Memory (Electronic or Otherwise).” Leonardo, vol. 23, no. 4, 1990, pp. 397-99, doi: $10.2307 / 1575342$.

Foucault, Michel. The Order of Things: An Archaeology of the Human Sciences. Routledge, 1994.

Fuller, Buckminster. “Buckminster Fuller.” Perspecta, vol. 1, 1952, pp. 28-37, doi: 10.2307/1566844.

Fuller, Buckminster, and Kiyoshi Kuromiya. Critical Path. 2nd ed., St. Martin's Griffin, 1982.

Fuller, Buckminster. Operating Manual for Spaceship Earth. Southern Illinois University Press, 1969.

Fuller, Buckminster, and Jaime Snyder. Utopia or Oblivion: The Prospects for Humanity. Lars Müller Publishers, 2008.

"Fragile Planet: Earth's Place in the Universe - Fulldome Show." Fulldome Database, 2008, www.fddb. org/fulldome-shows/fragile-planet-earths-place-in-the-universe. Accessed 16 May 2020.

Fraction. “ENTROPIA.” Vimeo, uploaded by Fraction, 6 May 2015, vimeo.com/129903465. Accessed 16 May 2020.

Griffiths, Alison. Shivers Down Your Spine: Cinema, Museums, and the Immersive View (Film and Culture Series). Columbia University Press, 2008.

Jasanoff, Sheila. “Technologies of Humility.” Nature, vol. 450, no. 7166, 2007, p. 33, doi: 10.1038/ 450033a.

Interaction, Tangible. “OH! For Science World.” Vimeo, uploaded by Tangible Interaction, 30 Oct. 2020, vimeo.com/258193854. Accessed 16 May 2020.

Jones, Caroline, et al. Sensorium: Embodied Experience, Technology, and Contemporary Art. Illustrated, MIT Press, 2006.

Jean, Hayley. "Connecting Art and Science: An Artist's Perspective on Environmental Sustainability”. Environmental Studies, Electronic Thesis Collection, 2019.

Lambert, Nick. "Domes and Creativity: A Historical Exploration.” Digital Creativity, vol. 23, no. 1, 2012, pp. 5-29, doi: 10.1080/14626268.2012.664821.

Loukissas, Y. A. “Software Takes Command.” Journal of Design History, vol. 28, no. 1, 2014, pp. 10708, doi: 10.1093/jdh/epu017. 
Mackinnon-Day, Patricia. "An Artist's Anthropological Approach to Sustainability.” International Journal of Art \& Design Education, vol. 35, no. 3, 2016, pp. 307-15, doi: 10.1111/jade.12122.

Manovich, Lev. Software Takes Command(International Texts in Critical Media Aesthetics). Illustrated, Bloomsbury Academic, 2013.

Mooney, H. A., et al. "Evolution of Natural and Social Science Interactions in Global Change Research Programs.” Proceedings of the National Academy of Sciences, vol. 110, no. Supplement_13665-72, 2013, doi: 10.1073/pnas.1107484110.

Marché, Jordan D. Theaters of Time and Space: American Planetaria, 1930-1970. Rutgers University Press, 2005.

Meade, Michael. "Creative Ecology: Art's Role in Addressing Environmental and Sustainability Issues in Australia”. Independent Study Project (ISP) Collection, 2008, https://digitalcollections.sit.edu/ isp_collection/583 Accessed 16 June 2020.

"Mission Earth - Fulldome Show." Fulldome Database, 2019, www.fddb.org/fulldome-shows/missionearth. Accessed 16 May 2020.

Mossessian, Claude. "Miguel CHEVALIER In / Out - Paradis Artificiels 2017 Domaine de Chaumont Sur Loire.” Vimeo, uploaded by Claude Mossessian, 9 Apr. 2017, vimeo.com/212438994. Accessed 16 May 2020.

McConville, David. On the Evolution of the Heavenly Spheres: An Enactive Approach to Cosmography. University of Plymouth Research Theses, 2014.

MySquare, Francois Moncarey. "KosmoVision-MySquare-2019." Vimeo, uploaded by Francois Moncarey, 1 Feb. 2020, vimeo.com/388964788. Accessed 16 May 2020.

“Our Living Climate - Fulldome Show.” Fulldome Database, 2019, www.fddb.org/fulldome-shows/ our-living-climate. Accessed 16 May 2020.

Pasternack, Alex. “This Amazing New Planetarium Show Is like Google Earth for the Universe.” Fast Company, 31 Jan. 2020, www.fastcompany.com/90453808/this-amazing-new-planetarium-showis-like-google-earth-for-the-universe. Accessed 16 May 2020.

Pluriel, Kondition. "Future Room - a $360^{\circ}$ Fulldome Installation.” Vimeo, uploaded by kondition pluriel, 27 Jan. 2020, vimeo.com/387432819. Accessed 16 May 2020.

Renn, Jürgen. The Evolution of Knowledge: Rethinking Science for the Anthropocene. Princeton University Press, 2020.

Reno, Joshua. “Earths.” Anthropocene Unseen, 2020, pp. 133-37, doi: 10.2307/j.ctv11hptbw.23.

Saldanha, Arun, and Hannah Stark. "A New Earth: Deleuze and Guattari in the Anthropocene.” Deleuze Studies, vol. 10, no. 4, 2016, pp. 427-39, doi: 10.3366/dls.2016.0237.

Rispoli, Giulia, and Jacques Grinevald. "Vladimir Vernadsky and the Co-Evolution of the Biosphere, the Noosphere, and the Technosphere.” Technosphere Magazine, 20 June 2018, technospheremagazine.hkw.de/p/Vladimir-Vernadsky-and-the-Co-evolution-of-the-Biosphere-the-Noosphereand-the-Technosphere-nuJGbW9KPxrREPxXxz95hr. Accessed 16 May 2020.

Russill, Chris. "Ecomedia." Earth Imaging. Photograph, Pixel, Program, edited by Stephen Rust et al., 2015, doi: 10.4324/9781315769820. 
Schaumberg, Erica. “Carved Ivory Puzzle Balls - Wonders of Nature and Artifice.” Wonders of Nature and Artifice, 2017, wonder-cabinet.sites.gettysburg.edu/2017/cabinet/carved-ivory-puzzle-balls. Accessed 16 May 2020.

Schneider, Laurel. Beyond Monotheism: A Theology of Multiplicity. 1st ed., Routledge, 2007.

Shah, Nadiya. "The Disenchantment of Modernity and The Re-Enchantment of the Cosmos." Nadiya Shah, Nadiya Shah, 7 June 2019, nadiyashah.com/the-disenchantment-of-modernity-and-the-reenchantment-of-the-cosmos. Accessed 16 May 2020.

Steffen, Will, et al. "The Emergence and Evolution of Earth System Science.” Nature Reviews Earth \& Environment, vol. 1, no. 1, 2020, pp. 54-63, doi: 10.1038/s43017-019-0005-6.

“Super Salvador - Fulldome Show." Fulldome Database, 2019, www.fddb.org/fulldome-shows/supersalvador. Accessed 16 May 2020.

“The Trouble with Space Junk | Flandrau Science Center \& Planetarium.” FLANDRAU Science Center and Planetarium, The Arizona Board of Regents on behalf of The University of Arizona, 10 Feb. 2019, flandrau.org/news/2019/02/trouble-space-junk. Accessed 16 May 2020.

"The Overview Effect." Overview Institute, The Overview Institute of Australia, www.overview instituteaustralia.org/the-overview-effect. Accessed 16 May 2020.

Thornes, John E. “A Rough Guide to Environmental Art.” Annual Review of Environment and Resources, vol. 33, no. 1, 2008, pp. 391-411, doi: 10.1146/annurev.environ.31.042605.134920.

Trubshaw, Don. “'The Re-Enchantment of the World' as Social Theory and Critique.” The Axiological Perspective, Don Trubshaw, 13 June 2017, theaxiologicalperspective.wordpress.com/2017/06/13/ the-re-enchantment-of-the-world-as-social-theory-and-critique. Accessed 16 May 2020.

Turner, Edith. Communitas: The Anthropology of Collective Joy. Palgrave Macmillan, 2011.

Turner, V. "Liminal to Liminoid, in Play, Flow, and Ritual: An Essay in Comparative Symbology." Rice Institute Pamphlet - Rice University Studies 60, pp. 123-64, 1974.

Vernadskiř, Vladimir Ivanovich, and Vernadsky. Scientific Thought as a Planetary Phenomenon. Nongovernmental Ecological V.I. Vernadsky Foundation, 1997.

Weibel, Peter. "The Post-media Condition”. AAVV, Postmedia Condition, cat., Centro Cultural Conde Duque, Madrid, 2006.

Wilson, G., and W. Huntress. "Mission to Planet Earth." Global and Planetary Change, vol. 4, no. 1-3, 1991, pp. 317-28, doi: 10.1016/0921-8181(91)90113-b.

Yu, Ka Chun. “Data to Dome 06/14 - International Planetarium Society, Inc.” International Planetarium Society, International Planetarium Society, 2014, www.ips-planetarium.org/page/ DataDome0614. Accessed 16 May 2020.

Zalasiewicz, Jan, et al. "Scale and Diversity of the Physical Technosphere: A Geological Perspective." The Anthropocene Review, vol. 4, no. 1, 2016, pp. 9-22, doi: 10.1177/2053019616677743.

Zylinska, Joanna. Nonbuman Photography. 1st ed., The MIT Press, 2017. 